\title{
What effect can rhythmic finger tapping have on the phonological similarity effect?
}

\author{
SATORU SAITO \\ Kyoto University, Kyoto, Japan
}

\begin{abstract}
The effects of rhythmic finger tapping on the phonological similarity effect were investigated in two experiments. In both, subjects were tested for serial recall of visually presented letter sequences that were either phonologically similar or dissimilar. The letter sequences had to be remembered under three tapping conditions: right-hand tapping, left-hand tapping, and a no-tapping control. Experiment 1 showed clear phonological similarity effects in both the control and the left-hand tapping conditions, but not in the right-hand tapping condition, when recall responses were written with the right hand. When the number of tapping practice trials was fixed at two and recall was vocal in Experiment 2, the phonological similarity effect was eliminated in both the right-hand and the left-hand tapping conditions. These results suggest that some form of speech motor programs played an important role in serial recall.
\end{abstract}

Over the past 30 years, it has been shown that there is a close relationship between phonological coding and short-term memory. Conrad and Hull (1964) showed, for example, that the immediate memory of sequences of letters was impaired when the letters concerned were phonologically similar. This is the "phonological similarity effect." The pattern of this effect indicates the importance of the role played by phonological coding of letters in short-term memory.

When the materials are presented visually, the phonological similarity effect is abolished by articulatory suppression, a method in which the subject is required to continuously articulate some irrelevant speech sound such as the word " $h i-y a$ " (Richardson, Greaves, \& Smith, 1980) or "the" (Murray, 1968; Wilding \& Mohindra, 1980), or the ordinal counting of digits (Besner \& Davelaar, 1982; Peterson \& Johnson, 1971). With auditory presentation, however, the phonological similarity effect withstands the articulatory suppression (Levy, 1971; Murray, 1968; Peterson \& Johnson, 1971).

These results have been explained by the concept of an articulatory loop that is a part of working memory (Baddeley, 1986). The articulatory loop consists of a phonological store and an articulatory control process. The phonological similarity effect is said to be due to the operation of the passive phonological store. Auditory information has direct access to this store, but visual information has

The data from the male subjects in Experiment 1 were presented at the 22nd International Congress of Applied Psychology, Kyoto, Japan, July 1990. I would like to thank Noboru Sakano and Masuo Koyasu of Kyoto University for their valuable comments on this study. In addition, I would like to thank Robert Proctor and three reviewers for helpful suggestions concerning revision. Correspondence should be addressed to S. Saito, Research Center for School Education, Naruto University of Education, Takashima, Naruto-shi, 772 Japan.

- Accepted by previous editor, Margaret Jean Intons-Peterson access only via the articulatory control process. The articulatory control process serves to allow visually presented material to be phonologically coded (Baddeley, 1986). Hence, in visual presentation, if the operation of the articulatory control process is prevented by articulatory suppression, the phonological similarity effect disappears.

What component of articulatory suppression interferes with the articulatory control process? Baddeley (1990) suggested that the articulatory control process does not depend on peripheral speech muscles for its operation, but presumably on some form of motor program at a central level. This was confirmed by examining the memory performance of dysarthric subjects-that is, patients whose capacity to control their articulatory muscles was reduced by brain damage. The fact that the patients showed, for example, in spite of the damage they had suffered, the phonological similarity effect with visual presentation indicates that they could operate the articulatory control process without feedback from organs for articulation (Baddeley \& Wilson, 1985). Therefore, the operation of the articulatory control process must be disturbed by articulatory suppression through preemption or distraction of speech motor programs, not of peripheral speech muscles.

This explanation is also consistent with the evidence from patients with apraxia of speech, who show a disruption in the programming that positions their speech muscles to produce phonemes. Waters, Rochon, and Caplan (1992) found that these patients showed the phonological similarity effect for auditory materials but not for visually presented ones. This pattern is similar to that seen in normals tested under conditions of articulatory suppression.

The purpose of the present study was to confirm that the activity of the articulatory control process involves the operation of speech motor programs. It is possible to examine this hypothesis by using a method that distracts speech motor programs without requiring articulatory movements. Studies of inner speech have used such an 
interference method, in which covert articulation is suppressed by distraction of the running of speech motor programs. For instance, in Zhinkin's studies from 1960 and 1964 (cited in Sokolov, 1966/1972), while solving mental problems of various kinds, subjects were required to tap one of their hands in a certain rhythm. The steady tapping rhythm was expected to upset the intermittent rhythm of speech movements (Sokolov, 1966/1972). The present experiments were designed to investigate the effects of rhythmic finger tapping on the phonological similarity effect.

According to the working memory model, however, tapping does not affect the operation of the articulatory loop. Furthermore, tapping at the same rate as articulatory suppression is considered to be a control condition for evaluating articulatory suppression (Baddeley, 1990). Indeed, some reports have suggested that such tapping does not affect the performance of reading (Baddeley, Eldridge, \& Lewis, 1981) and counting (Logie \& Baddeley, 1987 ) as much as articulatory suppression does.

There may be two reasons why the tapping task in the preceding studies had little or no effect on the articulatory loop. First, tapping at the same rate as articulatory suppression is simple, so it should not distract the running of speech motor programs. Hence, in the present study, a tapping task that had a somewhat complex rhythm was used. Second, the tapping task used in previous studies was performed with the nondominant hand. It is known that in the case of right-handed subjects, tapping with the right hand is more effective at interfering with performance of a verbal task than is tapping with the left hand (e.g., Friedman, Polson, \& Dafoe, 1988). Therefore, the tapping hand was one of the factors considered in the present study. Subjects were tested for serial recall of visually presented letter sequences, which were either similar or dissimilar phonologically. Each block of the letter sequences was to be remembered under three tapping conditions: the right-hand and left-hand tapping conditions, and a control condition in which the subject was not required to perform the tapping task.

\section{EXPERIMENT 1}

Two hypotheses regarding the influence of rhythmic tapping on the phonological similarity effect can be generated. The main one is that the phonological similarity effect should be abolished by the complex rhythmic tapping, which should distract from the running of speech motor programs. This prediction is based on the view that the phonological coding of the materials presented visually depends on the activity of speech motor programs. The second hypothesis is concerned with differences between the tapping hands. Most explanations concerning hand differentiation depend on the neuropsychological assumptions that (1) the manual activity of each hand is programmed primarily by the contralateral cerebral hemisphere, (2) verbalization in most right-handed people involves primarily the left cerebral hemisphere, and (3) two independent tasks will interfere with each other more when they involve the same cerebral hemisphere than when they involve different cerebral hemispheres (Hellige \& Longstreth, 1981). It seems that the operation of the articulatory loop may be based on the left-hemisphere functions, because the loop is assumed to be responsible for the manipulation of speech-based information. Hence, it is likely that right-hand tapping affects the phonological similarity effect more than left-hand tapping does.

\section{Method}

Subjects. The subjects were 16 male and 16 female undergraduate psychology students at Kyoto University. All were right-handed. The average age was 22.4 years for the males and 20.0 years for the females. They were non-native speakers of English, but were familiar with the alphabet letters used in this experiment as the tobe-remembered materials.

Design. The experiment used a two (phonological similarity: dissimilar and similar) $\times$ three (tapping condition: control, and righthand and left-hand tapping) factorial design. All factors were manipulated within subjects

Namely, all subjects were submitted to an experimental session composed of six blocks as follows: dissimilar-control (DC), dissimilar-right (DR), dissimilar-left (DL), similar-control (SC), similar-right (SR), and similar-left (SL). Each block had two practice trials that were not scored and then five test trials that were scored.

There were four orders of blocks: DC-SC-DR-SR-DL-SL, DC-SC-DL-SL-DR-SR, SC-DC-SR-DR-SL-DL, and SC-DCSL-DL-SR-DR. Each of these orders was presented to and performed by 8 subjects ( 4 males and 4 females).

Material and Procedure. In the right-hand and left-hand tapping conditions (dual-task conditions), the memory task and the rhythmical finger tapping had to be performed simultaneously. The tapping task required the subjects to listen to a sequence of a rhythm and to tap a button in synchronization with this rhythm. In the control condition, the subjects performed only the immediate memory task, hearing the same rhythm as that used in the dual-task conditions but without the tapping task.

Memory task. On each trial, the subjects were shown a sequence of six letters one at a time, either $B, C, D, G, P, T$ (phonologically similar set) or $\mathrm{K}, J, M, Q, R, Y$ (dissimilar set), in a different order each time. The subjects' task was to remember the order of the letters. Following an auditory warning signal, the letters were presented sequentially for $500 \mathrm{msec}$ each on a CRT slaved to a personal computer (NEC; PC-9801m). A 5-sec unfilled delay followed the termination of the last letter, and the end of the delay was indicated by visually and auditorily signaling the start of the recall period. The visual signal was a "?" on the CRT and the auditory signal was a 50-msec "beep" tone from the personal computer. Then, subjects picked up a pen and performed written serial recall with the right hand. The subjects wrote from left to right on a recall sheet that contained six blank boxes. They were allowed to retrace leftward to change an answer, but they were not allowed to fill in a blank on the right side until all of the preceding blanks to its left were filled. No empty blanks were allowed to remain unfilled. When the subjects finished a recall, then, for the following trial, the recall sheet was replaced by the new sheet.

Tapping task. The tapping task required the subject to tap a button of Microsoft mouse with his/her index finger, in synchronization with a sequence of a rhythm that a personal computer (Epson; PC$286 \mathrm{LS}$ ) auditorily indicated (tone frequency, $440 \mathrm{~Hz}$ ). This rhythm pattern was composed of 5 notes with 2 syncopations per bar (see Figure 1); a bar had 4 beats (a beat $=400 \mathrm{msec}$ ). Tapping was continued till the "?" signal indicated the end of the retention interval. Consequently, the subject had 26 tappings ( 25 intervals) to be counted in one trial. The tapping intervals were recorded by the personal computer. If a tapping interval differed from the duration of the correct note by more than $50 \mathrm{msec}$, an error was counted. 


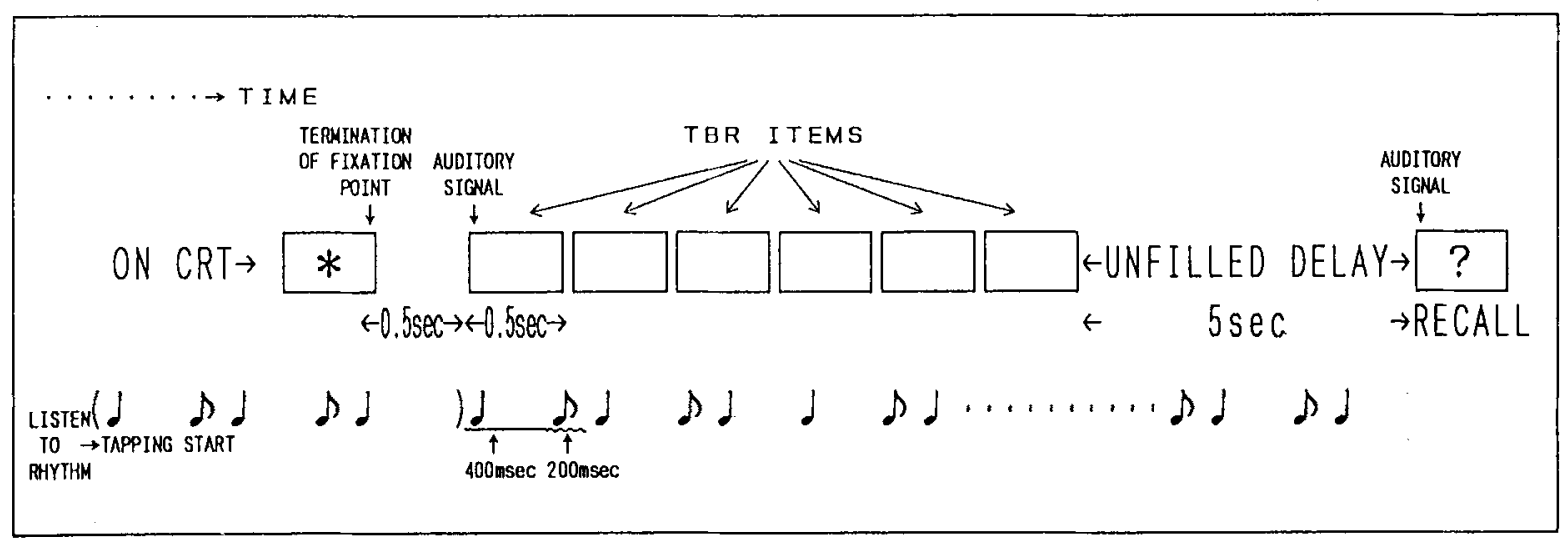

Figure 1. Task requirement in dual-task conditions.

The subjects practiced the rhythmical finger-tapping task alone with the hand assigned to the following block. The practice was repeated until no error was observed. Then the subject was engaged in the dual-task blocks.

Dual task. In the dual-task conditions, a trial consisted of the following stages (see Figure 1). First, in a period of $1,600 \mathrm{msec}$ (a bar), while hearing a pattern of the rhythm, the subjects were also prepared for the following tapping stage. Then, second, they started tapping the button in synchronization with the rhythm. They continued the tapping for a duration of 1,600 $\mathrm{msec}$. In the third stage, while the subjects was continuing the tapping task, a sequence of six letters were presented and a 5-sec delay followed the termination of the last letter. When recall was required, the subjects stopped tapping the rhythm. After that, they started to recall. Before the experimental session, the subjects were instructed that both the memory and the tapping task were important.

\section{Results}

Recall data. Letters were scored as correct if they were recalled in the same serial position in which they were presented. The data were initially analyzed with a three-way analysis of variance (ANOVA), with sex as a betweensubjects factor and phonological similarity and tapping as within-subject factors. Because all interactions with sex and a main effect of sex were not reliable [all $F \mathrm{~s}<1$ ], the following analyses were conducted collapsing across sex.

Figure 2 shows the proportion of correct responses as a function of phonological similarity and tapping. Clear phonological similarity effects were obtained in both the control and the left-hand tapping conditions, but not in the right-hand tapping condition. A two-way ANOVA, with phonological similarity and tapping, led to main effects of both phonological similarity $[F(1,31)=18.25$, $\left.M S_{\mathrm{e}}=.025, p<.001\right]$ and tapping condition $[F(2,62)=$ $\left.11.14, M S_{\mathrm{e}}=.020, p<.001\right]$. More important was the fact that the two-way interaction between phonological similarity and tapping condition was statistically significant $\left[F(2,62)=3.31, M S_{\mathrm{e}}=.013, p<.05\right]$.

Subsidiary analyses indicated that the dissimilar condition had an advantage over the similar condition in both the control and the left-hand conditions [respectively, $F(1,31)=15.69, M S_{\mathrm{e}}=.020, p<.01 ; F(1,31)=$ $\left.10.93, M S_{\mathrm{e}}=.020, p<.01\right]$. In contrast, the phonological similarity effect was not significant in the righthand tapping condition $[F(1,31)=1.71]$.

Tapping error. The average numbers of tapping errors per trial were as follows: 2.18 (right hand), and 1.93 (left hand) for the dissimilar conditions; and 2.12 (right hand), and 1.91 (left hand) for the similar conditions. A two-way ANOVA with phonological similarity and tapping hand was conducted. No significant difference was detected [all $F$ s $<2$ ].

\section{Discussion}

In this experiment, the phonological similarity effect occurred in the control condition and was also observed in the left-hand tapping condition. In contrast, the phonological similarity effect was perturbed in the right-hand tapping condition. The absence of a significant effect on tapping error indicates that the observed interference effects on the recall performance cannot be attributed to a tradeoff with the tapping performance. Consequently, the main hypothesis of Experiment 1, that the phonological similarity effect would be eliminated by rhythmic tapping, was verified.

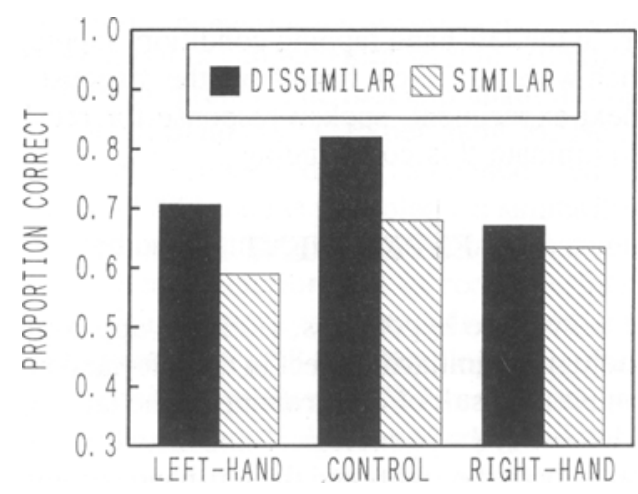

Figure 2. Proportion of correct recall in Experiment 1, as a function of phonological similarity (dissimilar and similar) and tapping (control, right-hand tapping, and left-hand tapping). 
An interpretation concerning the disappearance of the phonological similarity effect in the right-hand tapping condition is the following. The phonological similarity effect is a function of the passive phonological store, and visual material has access to this store via the articulatory control process, which involves the running of speech motor programs. Tapping of a rhythm can interfere with the running of the motor programs. Therefore, with the rhythmic tapping, the visual information of the materials cannot enter the phonological store. Hence, the phonological similarity effect disappears.

The interpretation of the results becomes more complex when one takes into consideration the reason why the tapping hand differentially affected the phonological similarity effect. This pattern of results is anticipated by the second hypothesis described in the introduction to this experiment. That is, the hand difference of this type may be explained by differences between functions of the left and the right cerebral hemispheres in general. However, before discussion of the hand difference in terms of the cerebral functions, two points had to be checked in the second experiment.

1. In the first experiment, practice of tapping was continued until no error was observed. The subjects may have needed more practice for left-hand tapping than for righthand tapping, because left-hand tapping may be more difficult for right-handed subjects. In fact, a supplemental experiment supported this point. Ten subjects engaged in the tapping practice with both the right and the left hands. They had 26 tappings ( 25 intervals) to be counted in one trial of tapping practice. The practice was repeated until no error was observed. The result showed that subjects required more practice to reach criterion with the left hand (6.0 trials) than with the right hand (2.4 trials). This difference was statistically significant $[F(1,9)=5.88$, $\left.M S_{\mathrm{e}}=11.022, p<.04\right]$. Thus, it was possibile that practice varied the cognitive processing. Hence, in the second experiment, the number of practice trials of tapping was fixed at two for both the left and the right hands.

2. In Experiment 1, written recall was performed with the right hand in all conditions. Thus, the right-hand tapping condition was one in which the tapping task was performed with the same hand as the hand of response. In contrast, in the left-hand tapping condition, tapping was performed with a different hand from the response hand. In the next experiment, spoken response for recall was used to eliminate this confounding.

\section{EXPERIMENT 2}

There were three hypotheses. (1) If the appearance of the phonological similarity effect in the left-hand tapping condition was a result of overtraining of the tapping task with the left hand, the phonological similarity effect should disappear in both the right- and the left-hand tapping conditions under the procedure including only two practice trials of the tapping task for both the left and the right hands. (2) If the phonological similarity effect disappeared in the right-hand tapping condition because the same hand was used for the recall and the tapping tasks, the phonological similarity effects should appear in both the rightand the left-hand tapping conditions under the procedure that uses spoken responses. (3) If the hand difference observed in the first experiment was due to the difference between functions of the left and the right cerebral hemispheres, Experiment 2 should show the same pattern of results as did Experiment 1. That is, the phonological similarity effect should occur in the left-hand tapping condition but not in the right-hand tapping condition.

\section{Method}

Subjects. The subjects were 16 undergraduate psychology students ( 6 males and 10 females). All were right-handed. The average age was 21.0 years old. They were non-native speakers of English, but they were familiar with the alphabet characters

Design, Material, and Procedure. The general procedural details for this experiment were similar to those in Experiment 1, except for the following points. (1) Only two trials were used for tapping practice. During this practice period, tapping error was scored as that of the single-task condition for tapping. (2) Spoken recall was used. After starting the recall, the subjects responded orally from the first letter to the last one presented in the list. (3) To-beremembered materials for the dissimilar condition were changed, because $K, M$, and $Y$ have an intermediate amount of visual similarity. Instead of $K$ and $Y, H$ and $S$ were used. Thus, the letters for the dissimilar conditions were $H, J, M, Q, R$, and $S$. (4) The order of six blocks was randomized.

\section{Results}

Recall data. Letters were scored correct if they were recalled in the same serial position in which they were presented. Figure 3 shows the proportion of correct responses as a function of phonological similarity and tapping. As shown in Figure 3, a clear phonological similarity effect was obtained in the control condition, but not in the right-hand or the left-hand tapping condition. A twoway ANOVA, with phonological similarity and tapping, led to main effects of both phonological similarity $[F(1,15)$ $\left.=16.35, M S_{\mathrm{e}}=.012, p<.001\right]$ and tapping $[F(2,30)=$ $\left.16.90, M S_{\mathrm{e}}=.021, p<.001\right]$. More important was the

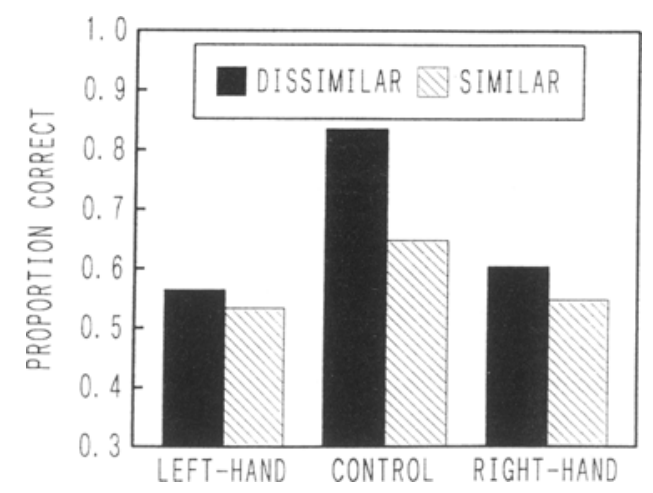

Figure 3. Proportion of correct recall in Experiment 2 as a function of phonological similarity (dissimilar and similar) and tapping (control, right-hand tapping, and left-hand tapping). 
fact that the two-way interaction between phonological similarity and tapping was statistically significant $[F(2,30)$ $\left.=3.38, M S_{\mathrm{e}}=.017, p<.05\right]$.

Subsidiary analyses indicated the advantage of the dissimilar condition over the similar in the control condition $\left[F(1,15)=26.36, M S_{\mathrm{e}}=.011, p<.001\right]$. In contrast, the phonological similarity effect was not significant in either the right-hand tapping or the left-hand tapping condition [respectively, $F(1,15)=2.09, F(1,15)=.34$ ]. The phonological similarity effect was virtually absent in both the right-hand tapping and the left-hand tapping conditions.

Tapping error. The average numbers of tapping errors per trial are presented in Table 1. A two-way ANOVA with learning condition (single task, dissimilar and similar conditions) and tapping hand (right and left hands) revealed an interaction $\left[F(2,30)=3.43, M S_{\mathrm{e}}=\right.$ $.700, p<.05$ ].

Subsidiary analyses confirmed the cause of the interaction between learning condition and tapping hand. A one-way analysis of the single-task condition showed a main effect of tapping hand $\left[F(1,15)=4.84, M S_{\mathrm{e}}=\right.$ $1.758, p<.05$ ]. In contrast, the analyses of both the dissimilar and the similar conditions indicated no main effect on tapping hand [respectively, $F(1,15)=.29 ; F(1,15)=$ $.01]$. Furthermore, the one-way analysis of the right-hand tapping condition led to a main effect of the learning condition $\left[F(2,30)=6.51, M S_{\mathrm{e}}=.837, p<.01\right]$. NewmanKeuls comparisons on the learning condition of the righthand tapping showed significant differences between single task and both the dissimilar and the similar conditions, but not between the dissimilar and the similar conditions. In the left-hand tapping condition, the effect of learning condition was not significant $[F(2,30)=.160]$.

\section{Discussion}

Results showed that the phonological similarity effect disappeared in both the right-hand tapping and the lefthand tapping conditions. Consequently, the first hypothesis of Experiment 2 was supported. That is, the appearance of the phonological similarity effect in the left-hand tapping condition of Experiment 1 may have been a result of overtraining of the tapping task with left hand.

Although significant effects of tapping hand were detected, the absence of a significant difference between the dissimilar and the similar conditions on tapping error indicates that the disappearance of the phonological similarity effect observed on the recall performance cannot be attributed to a tradeoff with the tapping performance.

Table 1

Mean Tapping Errors per Trial as a Function of Learning Condition (Single Task, Dissimilar, and Similar) and Tapping Hand

\begin{tabular}{ccc}
\hline Learning & \multicolumn{2}{c}{ Tapping Hand } \\
\cline { 2 - 3 } Condition & Left & Right \\
\hline Single task & 3.22 & 2.19 \\
Dissimilar & 3.39 & 3.14 \\
Similar & 3.23 & 3.25 \\
\hline
\end{tabular}

\section{GENERAL DISCUSSION}

In both experiments, subjects were tested for the serial recall of visually presented letter sequences, which were either similar or dissimilar phonologically, under three tapping conditions (no-tapping control, and right-hand and left-hand tapping). The results can be summarized as follows. In the first experiment, which used written recall with the right hand, the phonological similarity effect was observed in both the control and the left-hand tapping conditions but not in the right-hand tapping condition. However, when the number of practice trials in tapping task was fixed at two and vocal recall was used in the second experiment, the phonological similarity effect disappeared in both the right-hand tapping and the left-hand tapping conditions. That is, both the right-hand and the left-hand tapping affected the pattern of memory performance to the same extent.

These results of the two experiments indicate that a complex rhythmic tapping task can suppress the activity of the articulatory control process, as can articulatory suppression. The phonological similarity effect is a function of the passive phonological store, and visual material has access to this store through the articulatory control process. Tapping of the complex rhythm can suppress the running of the motor programs. Hence, with rhythmic tapping, the visual information about the materials cannot enter into the phonological store, and the phonological similarity effect therefore disappears.

Three points should be discussed here. First, why does a complex rhythmic tapping task interfere with the activity of the articulatory control process? Second, how does the tapping disturb the running of the speech motor programs? Third, why does the left-hand tapping condition in Experiment 1 show the phonological similarity effect?

The dysfunctioning of the articulatory control process with complex rhythmic tapping can be explained in either of two ways. (1) The attentional demand of the tapping task takes resources away from the articulatory loop or working memory system. Namely, for lack of resources, the articulatory control process cannot be used for the memory task. (2) The articulatory control process involves running the speech motor programs. Tapping of the complex rhythm can interfere with the running of the speech motor programs. The first explanation is opposed by the results of Saito (1993, Experiment 2). He investigated effects of memory updating, a method used by Morris and Jones (1990), on the phonological similarity effect. Although memory updating is an attention-demanding process that elicited a deterioration in recall performance, the phonological similarity effects still existed in the updating conditions (e.g., .58 for phonologically dissimilar, .41 for the similar condition). This result indicates that a lack of resources cannot eliminate the phonological similarity effect.

In contrast, the second explanation is well fitted to previous findings. As mentioned before, neuropsychological 
studies have shown that running the speech motor programs is essential for the functioning of the articulatory control process (Baddeley \& Wilson, 1985), and that dysfunctioning of the speech motor programs causes the disappearance of the phonological similarity effect (Waters et al., 1992). In sum, it seems reasonable to suppose that the articulatory control process involves running the speech motor programs and that tapping the complex rhythm can interfere with the running of the speech motor programs.

Articulatory suppression is a task that requires the subjects to utter irrelevant speech sounds, so that this process has complete overlap with speech motor programs and competes with them. In contrast, the motor programs used for the control of rhythmic finger tapping are not speech based. In this case, how does the rhythmic finger tapping interfere with the speech motor programs? There are at least two possible answers. One is that a part of the speech motor programs is used for performing the rhythmic tapping task. In other words, the speech motor programs partly overlap with the tapping motor programs. Thus, tapping motor programs compete with speech motor programs in taking sole possession of a part of the motor programs. The second is that, according to a model for overflow (Kinsbourne \& Hicks, 1978), the degree of interference is an inverse function of the "functional distance" between the cerebral control centers. Two cerebral control centers of these motor programs (for speech and tapping) are functionally close. Therefore, the tapping task interferes with the speech motor programs by motor overflow. Since the latter explanation expects the difference between the right-hand tapping and left-hand one, it is opposed by the results of Experiment 2 . Therefore, only the former seems viable.

One of the important problems in the present study was why the phonological similarity effect appeared in the lefthand tapping condition in Experiment 1 and not in Experiment 2. Of course, since Experiment 2 differed in two major ways from Experiment 1 (i.e., in the practice procedure and the change to vocal output), the different results for the left-hand tapping condition in the two experiments simply cannot be attributed to one of these factors. As indicated in the discussion of Experiment 2, however, the amount of practice may have been a factor that affected the phonological similarity effect. If so, why would practice of the tapping task lead to reinstatement of the phonological similarity effect? In other words, how can the speech motor programs operate under the condition of overtrained tapping? A possible answer to this question is the following. Complex rhythmic tapping used in the present study is a novel task to the subjects. They have no motor program for the tapping task at the first phase. Hence, other appropriate motor programs have to control the tapping activity until tapping motor programs become available. In oral language, important information consists not only of phonemes but also of prosody, which involves speech rhythms. The speech motor programs contain rhythmic aspects of speech, necessarily. For this reason, the speech motor programs can play the part of tapping motor programs. As mentioned earlier, a part of the speech motor programs is used for performing the rhythmic tapping task. With tapping practice, tapping motor programs become established and work independently of the speech motor programs. Thus, the speech motor programs can operate under the condition of tapping with the overtrained hand.

This explanation immediately raises the question of whether the effect of practice occurs independently between hands. Half the subjects were engaged in the left-hand tapping condition before performing right-hand tapping. If overpractice of left-hand tapping establishes tapping motor programs, these subjects in Experiment 1 should have the tapping motor programs before performing the righthand tapping task. In fact, they showed the phonological similarity effect in the right-hand tapping condition [.75 for the dissimilar condition and .66 for the similar condition; $F(1,15)=6.52, M S_{\mathrm{e}}=.009, p<.03$ ]. In contrast, the subjects who engaged in the right-hand tapping condition before performing left-hand tapping did not show the phonological similarity effect in the right-hand tapping condition $[.60$ for the dissimilar and .60 for the similar condition; $F(1,15)=.034]$. This suggests that the effect of practice does not occur independently between hands. That is, the motor programs for the left-hand tapping were used for the right-hand tapping task. It is consistent with the idea of Schmidt $(1975,1988)$ that a motor program should be considered as generalized. The idea of a generalized motor program is that a motor program for a particular class of action is stored in memory and that a unique pattern of activity will result if the program is executed (Schmidt, 1988, p. 240). For example, we can write some letters with the nondominant hand through the help of the generalized motor program.

In conclusion, the present results show that the phonological similarity effect was eliminated by rhythmic finger tapping. Some form of speech motor programs apparently play an important role in the immediate serial recall, and performing the complex rhythmic tapping task can suppress these motor programs. This suggests the importance of a motor component in working memory.

\section{REFERENCES}

Baddeley, A. D. (1986). Working memory. Oxford: Oxford University Press.

BadDEley, A. D. (1990). Human memory: Theory and practice. Hove, U.K.: Erlbaum.

Baddeley, A. D., Eldridge, M., Lewis, V. (1981). The role of subvocalization in reading. Quarterly Journal of Experimental Psychology, 33, 439-454.

BadDeley, A. D., \& Wilson, B. (1985). Phonological coding and shortterm memory in patients without speech. Journal of Memory \& Language, 24, 490-502.

Besner, D., \& DavelaAr, E. (1982). Basic processes in reading: Two phonological codes. Canadian Journal of Psychology, 36, 701-711.

Conrad, R., \& Hull, A. J. (1964). Information, acoustic confusion and memory span. British Journal of Psychology, 55, 429-432.

Friedman, A., Polson, M. C., \& Dafoe, C. G. (1988). Dividing attention between the hand and head: Performance trade-offs between rapid finger tapping and verbal memory. Joumal of Experimental Psychology: Human Perception \& Performance, 14, 60-68. 
Hellige, J. B., \& Longstreth, L. E. (1981). Effects of concurrent hemispheric-specific activity on unimanual tapping rate. Neuropsychologia, 19, 395-405.

Kinsbourne, M., \& Hicks, R. E. (1978). Functional cerebral space A model for overflow, transfer and interference effects in human performance. A tutorial review. In J. Requin (Ed.), Attention and performance VII (pp. 345-362). Hillsdale, NJ: Erlbaum.

LEVY, B. A. (1971). Role of articulation in auditory and visual shortterm memory. Journal of Verbal Learning \& Verbal Behavior, 10 , 123-132.

LogIE, R. H. , \& BADDElEY, A. D. (1987). Cognitive processes in counting. Joumal of Experimental Psychology: Learning, Memory, \& Cognition, 13, 310-326.

MorRIS, N., \& JoNES, D. M. (1990). Memory updating in working memory: The role of the central executive. British Journal of Psychology, 81, 111-121.

MurRay, D. J. (1968). Articulation and acoustic confusability in shortterm memory. Journal of Experimental Psychology, 21, 679-684.

Peterson, L. R., \& Johnson, S. T. (1971). Some effects of minimizing articulation short-term retention. Journal of Verbal Learning \& Verbal Behavior, 10, 346-354.

Richardson, J. T. E., Greaves, D. E., \& Smith, M. M. C. (1980).
Does articulatory suppression eliminate the phonemic similarity effect in short-term recall? Bulletin of the Psychonomic Society, 16, 417-420.

SaITo, S. (1993). Influence of articulatory suppression and memory updating on phonological similarity effect. Japanese Journal of Psychology, 64, 289-295. (In Japanese with English abstract)

SchmidT, R. A. (1975). A schema theory of discrete motor skill learning. Psychological Review, 82, 225-260.

SchmidT, R. A. (1988). Motor control and learning. Champaign, IL: Human Kinetics.

Sokouov, A. N. (1972). Inner speech and thought (G. T. Onischenko, Trans.). New York: Basic Books. (Original work published 1966)

Waters, G. S., Rochon, E., \& Caplan, D. (1992). The role of highlevel speech planning in rehearsal: Evidence from patients with apraxia of speech. Journal of Memory \& Language, 31, 54-73.

Wilding, J., \& Mohindra, N. (1980). Effects of subvocal suppression, articulating aloud and noise on sequence recall. British Journal of Psychology, 71, 247-261.

(Manuscript received September 23, 1992; revision accepted for publication June 22, 1993.) 